\title{
Mass-like Dieulafoy's lesion associated with advanced gastric cancer at the antrum of stomach: a case report and literature review
}

Hsi-Lan Huang ${ }^{1,2}$, Chi Yan Leung ${ }^{2}$ and Chien-Jui Cheng ${ }^{1 *}$

\begin{abstract}
Background: Dieulafoy's lesion, also known as a caliber-persistent artery, is a shallow, small, and rare lesion that occurs along the lesser curvature of proximal stomach. It is rare for a Dieulafoy's lesion to present as a mass-like lesion that coexists with gastric cancer. To our best knowledge, we report the first case and histopathological pictures of a mass-like Dieulafoy's lesion coexisting with advanced gastric cancer in the antrum of the stomach.

Case presentation: A 57-year-old female presented with a 6-month history of intermittent epigastric dull pain and dyspepsia. Subsequent upper gastrointestinal endoscopy revealed a friable mass that was located between the distal antrum and the pyloric ring. Biopsy revealed it to be an intestinal type adenocarcinoma. Subtotal gastrectomy was performed after neoadjuvant chemotherapy. Grossly, a large irregular plaque-like tumor lesion was noted at the anterior wall of the distal antrum and pylorus ring near the lesser curvature, measuring $5.6 \times 4.8 \times 1.0 \mathrm{~cm}$. Histopathological examination of the resected stomach revealed that the plaque-like lesion largely consisted of numerous abnormally large-caliber and tortuous arteries in the submucosa. The increased fibrosis of the submucosa resulted in the formation of elevated plaque. The intestinal type adenocarcinoma was noted to be largely confined to the mucosa layer, with focal submucosal and muscular propria involvement. The patient was discharged one week after the subtotal gastrectomy, and she was alive and well 17 months after discharge, with no major complications.

Conclusion: This is the first case of a mass-like Dieulafoy's lesion coexisting with advanced gastric cancer at the distal antrum area. This case highlights the possibility of life-threatening gastric bleeding after mucosal resection or biopsy that could be encountered by endoscopists.
\end{abstract}

Keywords: Advanced gastric cancer, Antrum, Dieulafoy's lesion, Caliber-persistent artery, Mass-like, Gastrointestinal bleeding

\section{Background}

Dieulafoy's lesion (DL), a rare arterial malformation of stomach, is an important cause of gastrointestinal bleeding. Extremely rare in the antrum, $60 \%$ of DL are confined to within $6 \mathrm{~cm}$ from the esophago-cardiac junction of stomach. Only 18 cases of stomach DL were ever reported to coexist with gastric cancer, and only 2 of the 18 cases were advanced gastric cancers (Table 1) [1-7].

\footnotetext{
* Correspondence: cjcheng@tmu.edu.tw

${ }^{1}$ Department of Pathology, Taipei Medical University Hospital, No.252, Wu

Hsing Street, Taipei City 110, Taiwan

Full list of author information is available at the end of the article
}

Although there is no definite pathophysiologic explanation regarding the association between $\mathrm{DL}$ and gastric cancer, discrete uniform pathologic descriptions have been reported in all cases - the location of DL constantly corresponded to the location of the gastric cancer $[5,8]$. To the best of our knowledge, no single English report describes a mass-like DL associated with advanced gastric carcinoma in the antrum. We report herein a case and histopathological pictures of a mass-like DL coexisting with advanced gastric cancer in the antrum.

(c) The Author(s). 2017 Open Access This article is distributed under the terms of the Creative Commons Attribution 4.0 International License (http://creativecommons.org/licenses/by/4.0/), which permits unrestricted use, distribution, and reproduction in any medium, provided you give appropriate credit to the original author(s) and the source, provide a link to the Creative Commons license, and indicate if changes were made. The Creative Commons Public Domain Dedication waiver (http://creativecommons.org/publicdomain/zero/1.0/) applies to the data made available in this article, unless otherwise stated. 


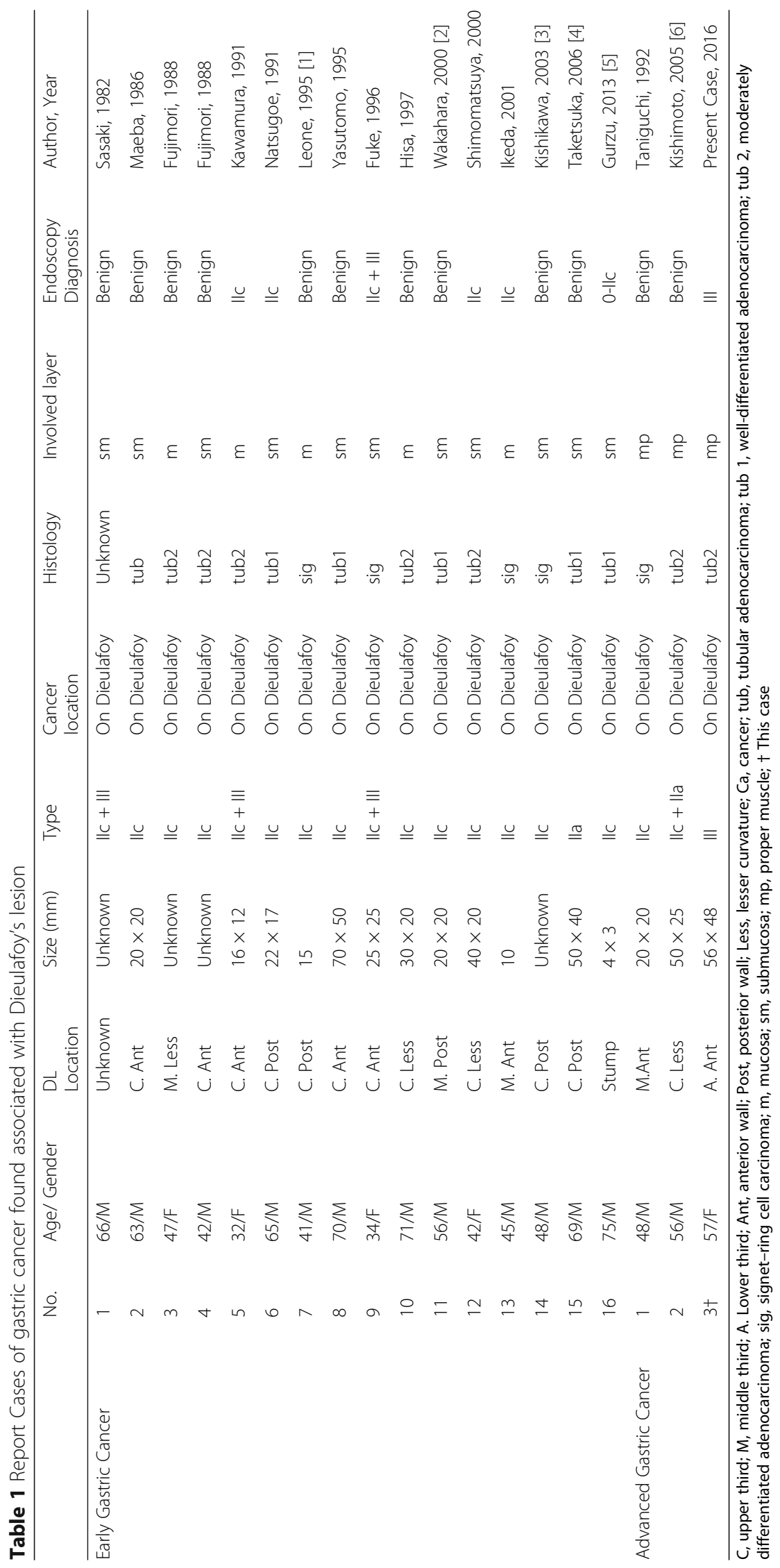




\section{Case presentation}

A 57-year-old female presented with a 6-month history of intermittent epigastric dull pain and dyspepsia. No gastrointestinal hemorrhage, use of non-steroidal antiinflammatory drugs, excessive alcohol ingestion, or chronic liver disease was reported. A positive fecal occult blood test was reported 3 months prior to admission. Hemoglobin and hematocrit were $10.1 \mathrm{~g} / \mathrm{dL}$ and $28 \%$, respectively, with an otherwise normal full blood picture. Subsequent upper gastrointestinal endoscopy revealed a friable mass which extended from the distal antrum to the pyloric ring. Routine biopsy showed the presence of intestinal type adenocarcinoma. Abdominal computerized tomography showed a tumor mass originating from the anterior wall of the antrum with a suspicion of lymph node metastasis. Subtotal gastrectomy was performed after neoadjuvant chemotherapy. Grossly, a large irregular plaque-like lesion, sized $5.6 \times$ $4.8 \times 1.0 \mathrm{~cm}$, was noted at the anterior wall of the distal antrum and pylorus ring near the lesser curvature (Fig. 1a). Postoperatively, the diagnosis of DL was made via histopathologic examination. At microscopy, numerous abnormally large-caliber tortuous arteries were identified in the submucosa with increased submucosal fibrosis, resulting in formation of an elevated plaque (Fig. 1b). The intestinal type adenocarcinoma was mostly confined to the mucosa (Fig. 1c), with focal submucosal and muscular propria involvement. Results of the serial section performed in the cancer and nontumor areas of the specimen are shown in Fig. 1d. The intima of the arteriolar wall exhibited focal thickening with ordinary elastic lamina (Fig. 2a, b). Two regional metastatic lymph nodes were reportedly found in the specimen. No lymphovascular invasion was identified. No Helicobacter pylori were seen in the regional nonneoplastic gastric mucosa. The regional non-neoplastic gastric mucosa revealed chronic gastritis with increased lymphocytes and plasma cells infiltrations. Focal intestinal metaplasia was noted. At the cancerous area, immunohistochemistry studies revealed diffusely positive for CEA (Fig. 2c) and an increased proliferative index in Ki-67 study (Fig. 2d). The patient was discharged 1 week later, and she was alive 17 months following surgery with regular follow-up.

\section{Discussion}

DL was first reported by Gallard in 1896 and was subsequently described more accurately by Georges Dieulafoy

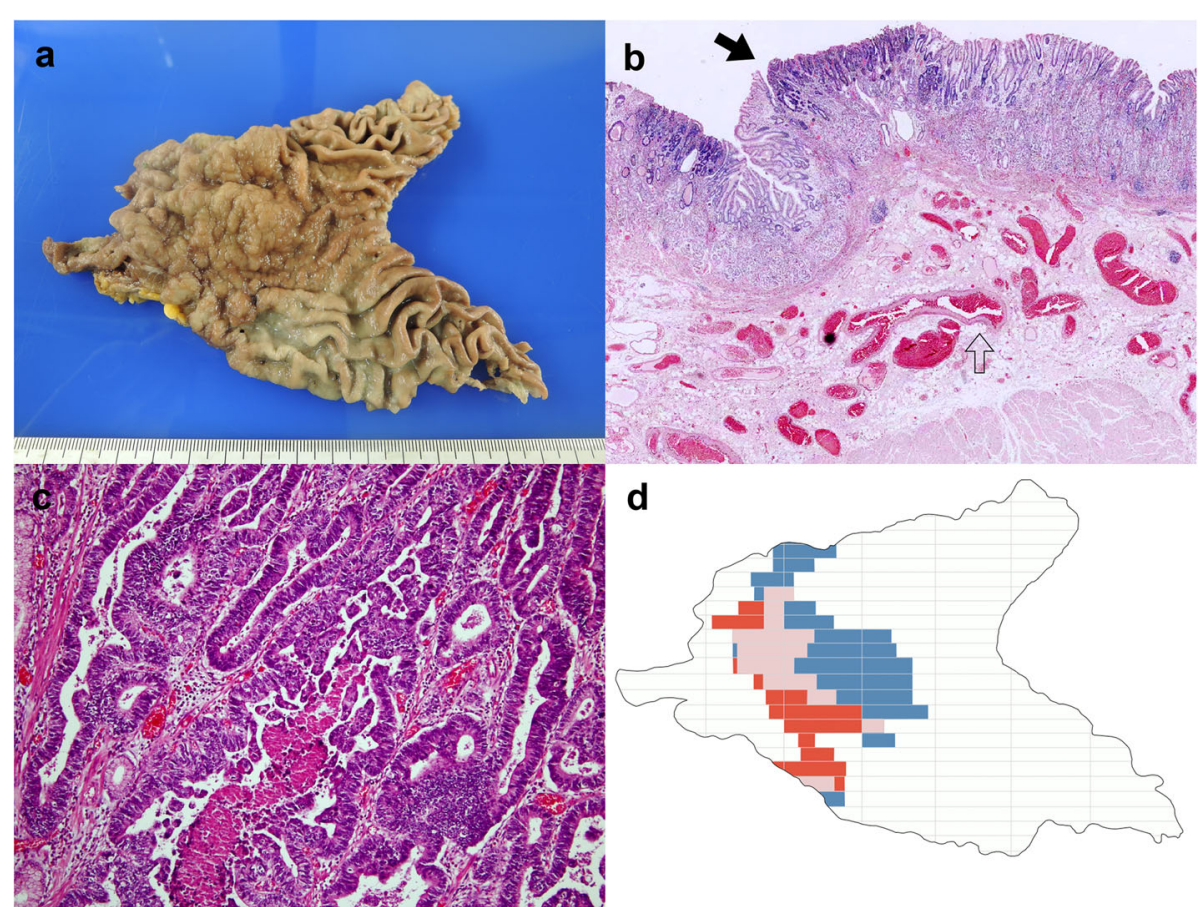

Fig. 1 a Gross features of the subtotal gastrectomy specimen. A protruded tumor was identified at the anterior wall of the distal antrum and pylorus ring near the lesser curvature. b Numerous abnormally large-caliber tortuous arteries in submucosa with increased submucosal fibrosis, resulting in formation of an elevated plaque. c The well differentiated adenocarcinoma was mostly noted within the mucosa (arrow). Beneath the adenocarcinoma, abnormally large-caliber tortuous arteries were identified in the submucosa with increased submucosal fibrosis (hollow arrow). d A schematic drawing of the resected specimen showed the distribution of the gastric cancer and Dieulafoy's lesions. The blue areas represented Dieulafoy's lesions without overlying gastric cancers. The red areas showed the gastric cancer without underlying Dieulafoy's lesion. The pink areas represented coexistence of gastric cancer and Dieulafoy's lesions (gastric cancer observed just above the Dieulafoy's lesions) 


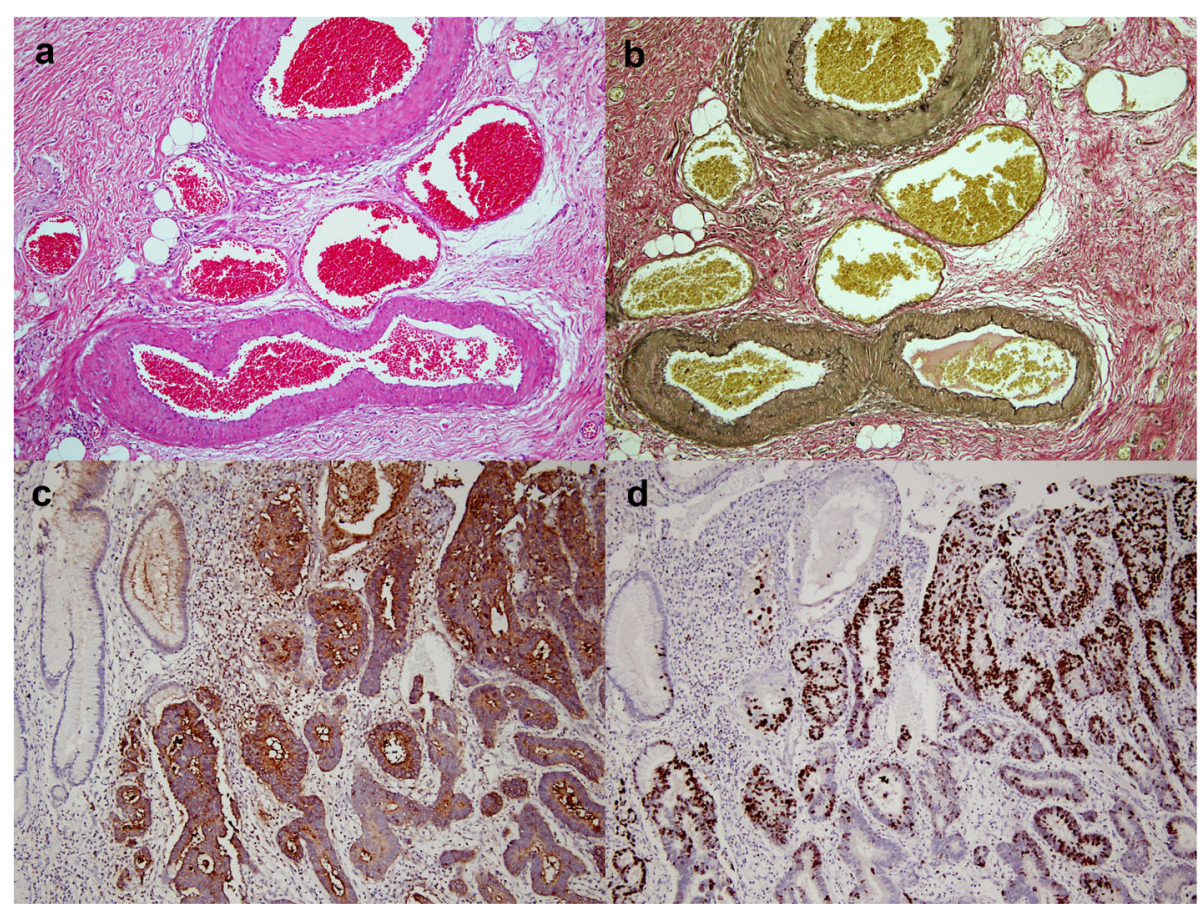

Fig. 2 a Histopathological findings of gastric caliber persistence or Dieulafoy's lesion. Oversized vascular submucosal clusters containing tortuous vessels could be seen within the submucosal layer. $\mathbf{b}$ Elastic stain demonstrated the persistent caliber arteries. At the cancerous area, immunohistochemistry studies revealed diffusely positive for CEA (c) and an increased proliferative index under Ki-67 study (d)

as "exulceratio simplex" in 1898 [9]. In modern times, DL has been interpreted as a congenital anomaly characterized by abnormally dilated submucosal arteries without aneurysm formation or intrinsic mural abnormality. Unlike normal arterial growth, arteries of DL remain persistently large in diameter even in distal branches. These distinctive histopathological patterns of DLs were described by Leone et al. as a "mixture of arterial and venous anomalies" [1]. The current case showed increased and proliferative clusters of vessels with arterial morphology in the submucosa beneath the tumor area, which is consistent with the histopathological picture of DL. Most DL (60\%) occur in the proximal stomach, $6 \mathrm{~cm}$ distal to the esophago-cardiac junction on the lesser curvature, and are supplied by the left gastric artery arising from the celiac trunk [7]. Twelve percent of DL are located at the distal antrum and are fed by the right gastric artery or an abnormal vascular anastomosis among the left and right gastric artery along the lesser curvature $[8,10]$. In our case, the lesion was located at the anterior wall of the distal antrum and pylorus ring near the lesser curvature. This atypical location could pose a diagnostic challenge to endoscopists. Awareness of possible adverse events during biopsy and mucosal resection that could be caused by the rare phenomenon of DL could be easily overlooked.
Upper gastrointestinal endoscopy is the major diagnostic modality for DL, and it is effective in up to $70 \%$ of patients. DL usually presents as small pigmented shallow protrusive lesions with mild erosion and no ulceration at endoscopy. Active spurting or oozing from a mucosal defect $<5 \mathrm{~mm}$ in diameter is often noted. The size of DL is about $10-15 \mathrm{~mm}$ in width and $5-10 \mathrm{~mm}$ in height. Angiography can be helpful in localizing the lesion, especially in the case of acute bleeding [8]. Masslike and papillae DL lesion in the stomach and small intestine have only been reported in two cases, and they were clinically and radiologically diagnosed as submucosal tumors instead [11, 12]. In our case, at endoscopy, the tumor appeared to be a plaque-like mass lesion with shallow ulceration and mucosal change similar to a typical gastric carcinoma, however the consistency of the tumor was soft and friable. The differential diagnosis at endoscopy included advanced gastric carcinoma and gastric lymphoma. This hybrid endoscopic finding suggests a diagnosis of mass-like DL.

The association between DL and gastric cancer is not fully elucidated. Taketsuka et al. hypothesized that repeated erosions and ulcers in the mucosa could be induced by circulation disturbances in vessels of DL, and active regeneration and dysplasia may further promote the process of carcinogenesis. This hypothesis is in line 
with the phenomenon that DL was located underneath the cancerous lesion in all reported cases [4]. Nonetheless, the prognosis of patients with gastric cancer concomitant with DL mainly depends on the pathologic stage of the gastric cancer.

Differential diagnosis of this case included gastric antral vascular ectasia (GAVE) and portal hypertensive gastropathy. GAVE accounts for up to $4 \%$ of all non-variceal upper gastrointestinal bleeding and is often associated with atrophic gastritis and pernicious anemia, which are known risk factors for gastric malignancy [13]. However, any association of GAVE and gastric cancer is reportedly rare [14]. Patients diagnosed are predominantly elderly females with mean age of 73 years, and they are usually associated with underlying chronic diseases, particularly cirrhosis (30\%) and autoimmune diseases (62\%) [15]. The characteristic endoscopic finding is "watermelon stomach" - alternating hyperemic streaks with normal mucosa radiating in a spoke-like fashion from the pylorus to the antrum. Characteristic histological pictures of GAVE include dilated capillaries and fibromuscular hyperplasia of the lamina propria, intravascular fibrin thrombi, and an increase in the mean cross-sectional area of the lumen in mucosal vessels, which were not observed in our case. Another differential diagnosis is portal hypertensive gastropathy, typically more prominent in the fundus or corpus and it is associated with liver cirrhosis.

This case highlights the possibility of mass-like DL coexisting with advanced gastric adenocarcinoma in the distal antrum. Also, our case alerts endoscopists to the risk of severe bleeding due to this unusual presentation, in view of recent preferences for the use of endoscopic modalities over surgical resection for DL treatment.

\section{Conclusion}

In summary, the association of mass-like DL and advanced gastric carcinoma at the distal antrum is an exceptionally rare condition. The postoperative diagnosis was due to the inability to distinguish these synchronous lesions during pre-operative gastroscopy. Hence, with the unique presentation and vascular architectures, this case highlights the possibility of life-threatening massive bleeding after biopsy and mucosal resection that could be encountered by endoscopists.

\section{Abbreviations}

DL: Dieulafoy's lesion; GAVE: Gastric antral vascular ectasia

\section{Acknowledgments}

We would like to thank Dr. Cheng-I Hsieh and Dr. Weu Wang (Taipei Medical University) for initial diagnosis and providing data. Manuscript was edited by MusesEdit. We wish to thank Dr. RSL Lo and Professor Colin A Graham, Emergency Medicine, Chinese University of Hong Kong for their advice on the manuscript.

\section{Funding}

There is no any financial support for this work.

\section{Availability of data and materials}

Data sharing not applicable to this article because no datasets were generated or analyzed during the current study.

\section{Authors' contributions}

$\mathrm{HLH}$ and CJC designed the study, collected clinical data and clinical interpretation, and performed the histological evaluation. HLH, CYL, and CJC participated in literature search, and drafted the manuscript. All authors read and approved the final manuscript.

\section{Authors' information}

Not applicable.

\section{Ethics approval and consent to participate}

The study had obtained the approval from ethics committee of Taipei Medical University Hospital (Institutional Review Board registration number: TMU-JIRB no. N201602033).

\section{Consent for publication}

Informed consent for publication was obtained from the patient.

\section{Competing interests}

The authors declare that they have no competing interests.

\section{Publisher's Note}

Springer Nature remains neutral with regard to jurisdictional claims in published maps and institutional affiliations.

\section{Author details}

'Department of Pathology, Taipei Medical University Hospital, No.252, Wu Hsing Street, Taipei City 110, Taiwan. ${ }^{2}$ Department of Global Health Policy, Medical Building No. 3, Hongo Campus, University of Tokyo, 7-3-1, Hongo, Bunkyo-ku, Tokyo, Japan.

Received: 26 April 2017 Accepted: 4 October 2017

Published online: 10 October 2017

\section{References}

1. Leone O, Zanelli M, Santini D, Minni F, Marrano D. Dieulafoy's disease associated with early gastric cancer. J Clin Pathol. 1995;48:267-70.

2. Wakahara M, Yasue S, Yasue T, Yasue M, Kuno T. A case of Ilc type early gastric cancer associated with massive gastrointestinal bleeding from Dieulafoy's ulcer. Nihon Gekakei Rengo Gakkaishi (J Jpn Coll Surg). 2000;25:647-51. (in Japanese)

3. Kishikawa H, Nishida J, Hosoe N, Nakano M, Morishita T, Masamura S, et al. Gastric cancer associated with Dieulafoy's lesion: case report. Gastrointest Endosc. 2003;57:969-72.

4. Taketsuka S, Kasama K, Kakihara Y, Horie K, Tagaya N, Kojima M, et al. Early gastric cancer located just above Dieulafoy's ulcer, with massive bleeding. Gastric Cancer. 2006:9:320-4

5. Gurzu S, Copotoiu C, Azamfirei L, Jung IA. Caliber persistent artery (Dieulafoy's esion) which is associated with an early-stage gastric stump cancer following a distal gastrectomy. J Clin Diagn Res. 2013;7:1717-9.

6. Kishimoto H, Sakakibara T, Yokoyama Y, Koyama S, Kuwana K, Tsukada K, et al. A case of gastric cancer associated with Dieulafoy's ulcer-like finding. Gastroenterol Endosc. 2005;47:313-7. (in Japanese)

7. Mikó TL, Thomázy VA. The caliber persistent artery of the stomach: a unifying approach to gastric aneurysm, Dieulafoy's lesion, and submucosal arterial malformation. Hum Pathol. 1988:19:914-21.

8. Nojkov B, Cappell MS. Gastrointestinal bleeding from Dieulafoy's lesion: clinical presentation, endoscopic findings, and endoscopic therapy. World J Gastrointest Endosc. 2015;7:295-307

9. Dieulafoy G. Exulceratio simplex. L'intervention chirurgicale dans les hematemeses foudroyantes consecutives a l'exulceration simple de l'estomac. Bull Acad Med. 1898;49:49-84.

10. Gurzu S, Copotoiu C, Molnar C, Azamfirei L, Jung I. Lethal gastric hemorrhage from a caliber-persistent artery of the antrum - a branch of the right gastric artery. Hippokratia. 2014;18:172-6.

11. Badwal S, Jain M, Rastogi A, Rawat A, Agrawal T. Dieulafoy disease of the stomach presenting as mass lesion-a case report. Indian J Pathol Microbiol. 2005:48:211-3. 
12. Kim JK, Jo BJ, Lee KM, Sun JS, Won JH, Kim MW, et al. Dieulafoy's lesion of jejunum: presenting small bowel mass and stricture. Yonsei Med J. 2005;46:445-7.

13. Calam J, Walker RJ. Antral vascular lesion, achlorhydria, and chronic gastrointestinal blood loss: response to steroids. Dig Dis Sci. 1980;25:236-9.

14. Berk T, Slemmer JR, Friedman LS. Gastric antral vascular ectasia associated with gastric carcinoma. Am J Gastroenterol. 1991;86:768-70.

15. Kar P, Mitra S, Resnick JM, Torbey CF. Gastric antral vascular ectasia: case report and review of the literature. Clin Med Res. 2013;11:80-5.

Submit your next manuscript to BioMed Central and we will help you at every step:

- We accept pre-submission inquiries

- Our selector tool helps you to find the most relevant journal

- We provide round the clock customer support

- Convenient online submission

- Thorough peer review

- Inclusion in PubMed and all major indexing services

- Maximum visibility for your research

Submit your manuscript at www.biomedcentral.com/submit
Biomed Central 